\title{
Using Blue Waters to Assess Tornadic Outbreak Forecast Capability by Lead Time
}

\author{
Caroline MacDonald \\ Department of Geosciences \\ Mississippi State, MS \\ cnm306@msstate.edu
}

\author{
Andrew Mercer \\ Department of Geosciences \\ Mississippi State, MS \\ a.mercer@msstate.edu
}

\begin{abstract}
Severe weather outbreaks come with many different hazards. One of the most commonly known and identifiable outbreaks are those with tornadoes involved. There has been some prior research on these events with respect to lead time, but shifts in model uncertainty by lead time has yet to be quantified formally. As such, in this study we assess tornado outbreak model uncertainty by lead time by assessing ensemble model precision for outbreak forecasts. This assessment was completed by first identifying five major tornado outbreak events and simulating the events using the Weather Research and Forecasting (WRF) model at 24, 48, 72, 96, and 120-hours lead time. A 10-member stochastically perturbed initial condition ensemble was generated for each lead time to quantify uncertainty associated with initialization errors at the varied lead times. Severe weather diagnostic variables derived from ensemble output were used to quantify ensemble uncertainty by lead time. After comparing moment statistics of several convective indices, the Energy Helicity Index (EHI), Significant Tornado Parameter (STP), and Supercell Composite Parameter (SCP) did the best job of characterizing the tornadic outbreaks at all lead times. There was good consistency between each case utilizing these three indices at all five lead times, suggesting outbreak model forecasting confidence may be able to extend up to 5 days for major outbreak events. These results will be useful for operational use by forecasters in forecast ability of tornadic events.
\end{abstract}

\section{Keywords}

Tornado outbreaks, stochastic initial condition perturbation, numerical weather prediction, ensemble forecasting

\section{INTRODUCTION}

Tornado outbreaks are one of the premier atmospheric phenomena that cause substantial damage across the United States each year. While considerable research has been performed on these outbreaks $[1,2,3]$, many questions remain regarding outbreak predictability and model uncertainty at increasing forecast lead time.

Permission to make digital or hard copies of all or part of this work for personal or classroom use is granted without fee provided that copies are not made or distributed for profit or commercial advantage and that copies bear this notice and the full citation on the first page. To copy otherwise, or republish, to post on servers or to redistribute to lists, requires prior specific permission and/or a fee. Copyright CJOCSE, a supported publication of the Shodor Education Foundation Inc.

(C) 2020 Journal of Computational Science Education DOI: https://doi.org/10.22369/issn.2153-4136/11/2/4
Previous research defines a tornado outbreak as ten or more tornadoes associated with a single synoptic-scale system and confined to a specific geographic area [1]. Importantly, $73 \%$ of deaths associated with historic tornadoes were associated with tornadic outbreak events [1]. On average, over 1,000 tornadoes occur across the United States each year (NCDC), a majority of which are associated with outbreaks. Additionally, tornadoes have the highest ten-year average fatality rate among weather related hazards from 2008 to 2017 [5].

Given the hazardous nature of tornadic events, forecasting of these events several days in advance is vital. Frequently, convective and kinematic indices are utilized to quantify outbreak characteristics and predict outbreak mode at varying lead times [3]. Many previous studies have studied short and medium-term outbreak model uncertainty using these indices $[3,6,7,8,9,10]$. Research has shown that there is a statistical drop off in forecast skill at lead times of 72 hours or longer [7], which is typical in most forecasting research. Keeping these limitations in mind, little research has been conducted looking beyond 72 hours in advance of tornadic outbreaks.

Recently, improvements in numerical weather predication models such as the Weather Research and Forecasting model (WRF) have resulted in improved forecasts of tornado outbreaks [9]. Such improvements result from prior knowledge and pattern recognition, and the availability of the WRF to reproduce historic major events has helped forecasters identify important meteorological details in these events. Thompson and Edwards [11] recognized that large scale outbreaks may not be as evident through numerical model output as previously thought. Their work showed that forecasts 12 to 24 hours in advance of the major 3 May 1999 tornado outbreak relied heavily on poor model output, which led to greater forecast uncertainty. An additional study, [12] relied on the WRF model to conduct similar case study research at varying lead times, noting forecast uncertainty increases with lead time. This suggests more work into model uncertainty is needed at both short and medium lead times. Clearly, a need for improved understanding of model uncertainty in tornado outbreak forecasts exists.

The objective of this research is to quantify uncertainty in tornado outbreak convective indices by lead time, which in turn will allow forecasters to have greater confidence when forecasting major tornadic outbreaks. Uncertainty quantification via ensemble modeling is a primary way to assess predictability as increasing ensemble spread relates directly to reduced model precision. Following the methods of previous research [13], five major tornado outbreaks were selected and WRF simulations conducted at lead times of 24, 48, 72, 96, and 120-hours were completed. Previous work suggests that short-term forecast uncertainty will be significantly less than medium-term (96 to 120 hour) forecasts, which is the primary hypothesis of this study. 
This student paper summarizes the purpose and results from research conducted through the Blue Waters Student Internship Program. Through this program, computational resources were utilized via the Blue Waters supercomputing facility. Section 2 will describe the data and methods utilized in this study as well as the computer and statistical modeling that aided in this research. Section 3 will discuss findings from this research at varying lead time intervals and the education impact related to this research. Additionally, this section will discuss the overall student goal and challenges associated with this study. The $4^{\text {th }}$ section will refer to reflections and discussion about what the student learned through this research and the computing knowledge the student gained through this program. Section 5 will highlight the summaries and draw conclusions from this study and highlight the needs of future research.

\section{DATA AND METHODS}

\subsection{Data}

As the primary goal of this research was to analyze the importance of lead time for tornadic severe weather outbreak forecasting, five major tornado outbreaks were selected from the Storm Event database [14] from the National Climatic Data Center. A major tornado outbreak was selected based on the number of tornadoes produced and overall damage extent of such tornadoes. Each of the five cases produced millions of dollars in damage, had several large, significant tornadoes, and frequently resulted in multiple deaths. Table 1 displays such statistics for each of the five outbreak cases [15-19].

Table 1. Displays of case date and statistical information for all five tornado outbreak cases utilized within this study

\begin{tabular}{|c|c|c|c|c|}
\hline Date & $\begin{array}{c}\text { Total \# of } \\
\text { Tornadoes }\end{array}$ & $\begin{array}{c}\text { EF/F 3+ } \\
\text { Tornadoes }\end{array}$ & Deaths & $\begin{array}{c}\text { Damage } \\
\text { (millions) }\end{array}$ \\
\hline $\begin{array}{c}10 \\
\text { April } \\
1979\end{array}$ & 29 & 5 & 50 & 63 \\
\hline $\begin{array}{c}28 \\
\text { March } \\
1984\end{array}$ & 24 & 12 & 57 & 600 \\
\hline $\begin{array}{c}31 \\
\text { May } \\
1985\end{array}$ & 43 & 14 & 89 & 600 \\
\hline $\begin{array}{c}3 \text { May } \\
1999\end{array}$ & 75 & 9 & 46 & 1500 \\
\hline $\begin{array}{c}17 \\
\text { June } \\
2010\end{array}$ & 74 & 8 & 4 & 100 \\
\hline
\end{tabular}

A robust database of three-dimensional meteorological analyses was required to complete WRF simulations on the five outbreaks. Given the spatial scale of the outbreak events and the dates of the events, data was obtained from a mesoscale reanalysis analysis dataset, the North American Regional Reanalysis [20]. The NARR are provided on a 32-km Lambert conformal North American grid with 3-hourly temporal resolution and 29 vertical levels, spanning 1979 to present. NARR fields were retained such that lead times of $24,48,72,96$, and 120 hours could be simulated using the WRF.

\subsection{Model Configuration and Simulations}

In this study, the WRF model was used to formulate the outbreak forecasts at varying lead times. The WRF is highly configurable, and in this study the configuration was kept consistent with previous research [13]. In particular, the WRF was configured using the WRF Single-Moment six class microphysics scheme [21], the Grell-Freitas convective scheme [22], the Yonsei University boundary layer scheme [23], the Rapid Radiative Transfer Model radiation scheme for longwave [24], and the Dudhia scheme [25] for shortwave radiation.

The WRF model (version 3.9) was utilized to run simulations of five tornadic outbreak events listed in Table 1. Since the primary goal of this research was to relate outbreak lead time intervals to forecast uncertainties as it relates to short and medium-term forecasting of tornadic outbreak events, each case was run at the five previously described lead time intervals. In this study, the simulation period was established by defining the end of the outbreak as 1200 UTC the day after the event. Lead times were then taken relative to this time, so that a 24-hour forecast started at 1200 UTC the day of the event, and so forth. All outbreaks had a peak activity time between 2100 UTC and 0600 UTC, meaning this simulation period sufficiently captured the active tornadic activity during the event.

Following the WRF simulations, severe weather diagnostics were computed on the simulation output, including Convective Available Potential Energy (CAPE), Significant Tornado Parameter (STP), Supercell Composite Parameter (SCP), Energy Helicity Index (EHI), and Storm Relative Helicity (SRH). CAPE and SRH are commonly utilized meteorological parameters for severe weather forecasting, where CAPE describes available thermodynamic potential energy (in $J / k g$ ) and SRH describes streamwise vorticity (in $\mathrm{m}^{2} / \mathrm{s}^{2}$ ) available to be tilted vertically and contribute to mesocyclone development. The remaining parameters were derived from a combination of SRH and CAPE. The three main equations discussed in further depth below are typically utilized with right-moving supercells, which is the predominant storm motion type within tornadic outbreaks utilized in this research.

EHI combines CAPE and SRH into a single convective index:

$$
\mathrm{EHI}=\frac{\mathrm{CAPE} * \mathrm{SRH}}{160,000 \frac{\mathrm{kg} \mathrm{s}^{2}}{\mathrm{Jm}^{2}}}(1)
$$

Larger values of EHI are thought to be indicative of environments supporting large tornadoes and tornadic supercells.

The STP is an index used to highlight the co-existence of ingredients favoring strong, damaging tornadoes [26]. This index was developed at the Storm Prediction Center to help identify meteorologically suitable regions for significant (EF2 or stronger) tornado activity [26]. The STP is given by:

$$
\operatorname{STP}=\left(\frac{\mathrm{mlCAPE}}{1500 \frac{\mathrm{J}}{\mathrm{kg}}}\right)\left(\frac{\mathrm{ESRH}}{150 \frac{\mathrm{m} 2}{\mathrm{~s} 2}}\right)\left(\frac{\mathrm{EBWD}}{12 \frac{\mathrm{m}}{\mathrm{s}}}\right)\left(\frac{2000-\mathrm{mlLCL}}{1000}\right)\left(\frac{\mathrm{mICINH}+200}{150}\right)
$$

Here, MLCAPE represents mixed-layer (ML) CAPE, a measure of CAPE derived from a parcel originating from a well-established mixed-layer. ESRH represents the SRH computed over the effective inflow layer [26]. EBWD represents the effective bulk wind difference (the wind vector difference between the top and bottom of the effective inflow layer). MLLCL represents the lifted 
condensation level of a parcel originating from the mixed layer. Smaller MLLCL values (ideally less than $1000 \mathrm{~m}$ ) are typical in tornadic environments. Finally, MLCINH represents the mixedlayer (ML) convective inhibition ( $\mathrm{CINH}$ ), which is needed to inhibit early-morning convection and create a buildup of boundary layer potential energy for afternoon explosive convective development.

The SCP is also a commonly used parameter in forecasting tornadic outbreak events, as it was developed to highlight areas where supercell development is favorable [26]. SCP was developed at the Storm Prediction Center in Norman, Oklahoma as well. The equation is as follows:

$$
S C P=\left(\frac{\mathrm{muCAPE}}{1000 \mathrm{~J} / \mathrm{kg}}\right)\left(\frac{\mathrm{ESRH}}{50 \frac{\mathrm{m} 2}{\mathrm{~S} 2}}\right)\left(\frac{\text { EBWD }}{10 \frac{\mathrm{m}}{\mathrm{s}}}\right)
$$

Here, MUCAPE represents the most-unstable (MU) convective available potential energy (CAPE), based on the most unstable parcel in the lowest $300 \mathrm{mb}$ of the atmosphere, and all other terms have been defined previously.

\subsection{Simulation Analysis}

To quantify forecast uncertainty, a spread in the forecast output was required. This spread was obtained by using the Stochastic Kinetic Energy Backscattering Scheme (SKEBS) [27] within the WRF configuration. The SKEBS provides initial condition perturbations to potential temperature and streamfunction values, essentially introducing random thermodynamic and kinematic noise into the model boundary conditions, which in turn creates model spread as the simulation progresses. Here, ten simulations for each case and lead time (a total of 250 simulations for all cases, perturbations, and lead times) were completed to assess WRF output uncertainty in the severe weather diagnostic fields. Uncertainty and distribution shape among the 10 perturbed simulations was identified through moment statistics (mean, variance, skewness, and kurtosis). The ten-member ensemble output was bootstrap-resampled 1000 times for each forecast timestep and moment statistics were formulated on the bootstrap replicates. The median bootstrap replicate for each moment statistic was then retained to assess ensemble member performance. The results are described below.

\section{DISCUSSION AND LESSONS LEARNED (EDUCATIONAL IMPACT)}

The primary goal of this study was to quantify uncertainty within tornado outbreak simulations with increasing lead time $(24,48,72$, 96 , and 120-hours). A multi-member stochastic perturbation initial condition ensemble was used to create simulation uncertainty, while outbreak characteristics were quantified using various severe weather indices. Overall, several indices stood out compared to other indices which aligned with previous research [7, 9]. SCP, STP, and EHI appear to be three of the convective indices that seemed to have the smallest uncertainty for on tornadic outbreak events at varying lead times, as indicated by the moment statistics calculated for the five test cases. Thermodynamic indices such as CAPE had a much larger spread of data as compared to these three indices. The ensembles and moment statists for these three variables appeared to be more consistent and uniform. While previous research $[3,6,7,8,9,10]$ has shown some variations in these indices as you increase lead time, this study seemed to show more consistent results at all five lead times. Figure 1 shows moment statistics from the May 3, 1999 tornado outbreak case utilizing the SCP guidance. For this case, peak outbreak time occurred at $00 \mathrm{Z}$ on May $4^{\text {th }}$ (denoted by the vertical bar in the figures). At this time, all five lead time mean intervals peaked at nearly the same SCP value and provided minimal positive skewness and some leptokurtic behavior, suggesting a gammadistribution type shape to the ensemble members that was consistent among all lead times.
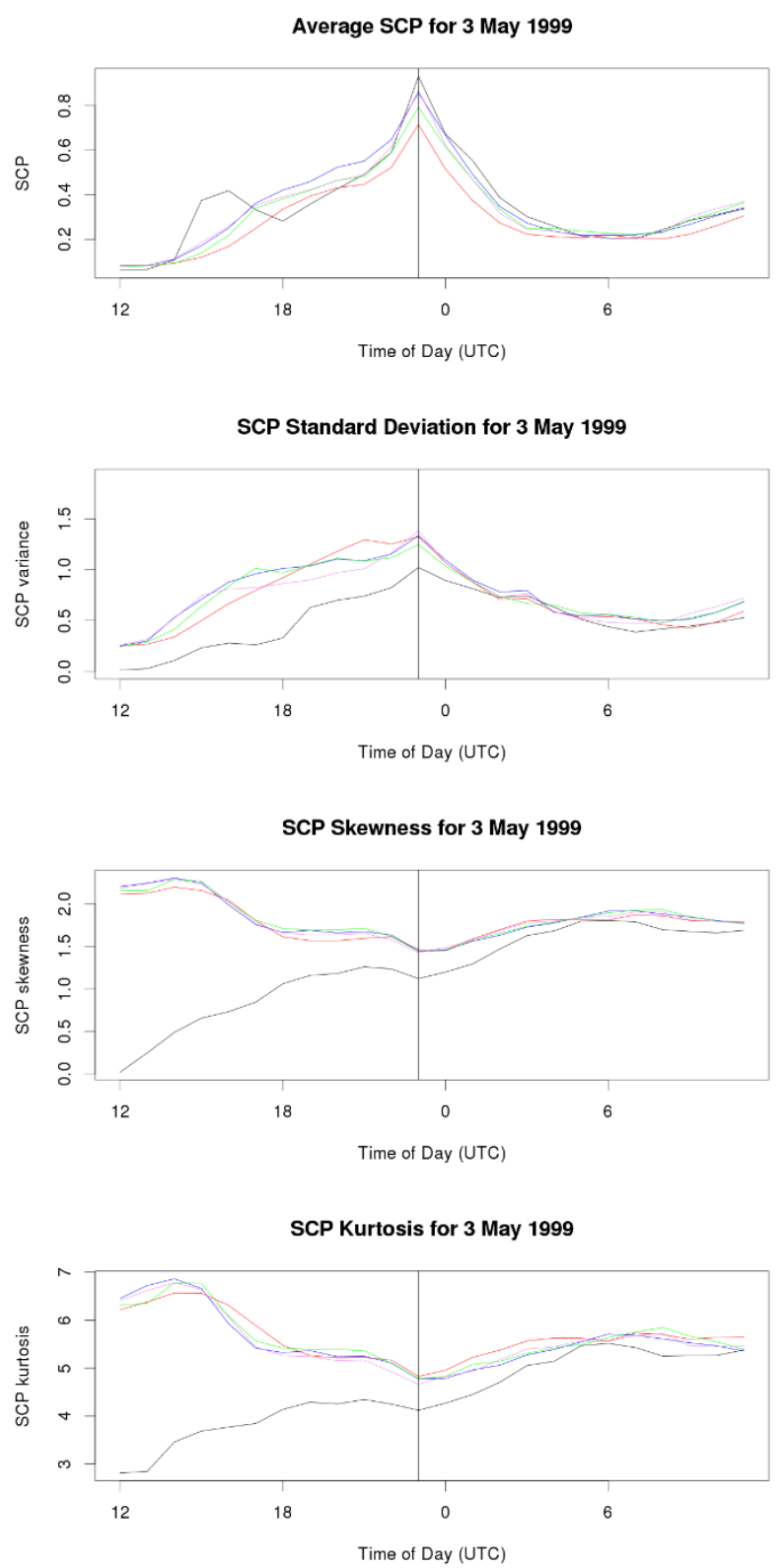

Figure 1. The four moment statistics (mean, standard deviation, skewness, and kurtosis) for the May 3, 1999 case are shown. All four moment statistics show similar results across all five lead time intervals. The only outlier is the 24hour lead time in the skewness and kurtosis plots, which could be due to the small amount of time as compared to the remaining four lead time intervals. Peak outbreak time is represented by the vertical black line.

These results suggest uncertainty remains consistent by lead time for these events, suggesting similar predictability at 120 hours exists at 24 hours. 
Kinematic indices such as SCP, STP, and EHI appear to be more beneficial in medium-term forecasting of tornadic outbreak events. These three indices had similar distribution characteristics at all five lead times and had the smallest spread, suggesting high ensemble precision in the kinematic fields, as shown in Figure 2.
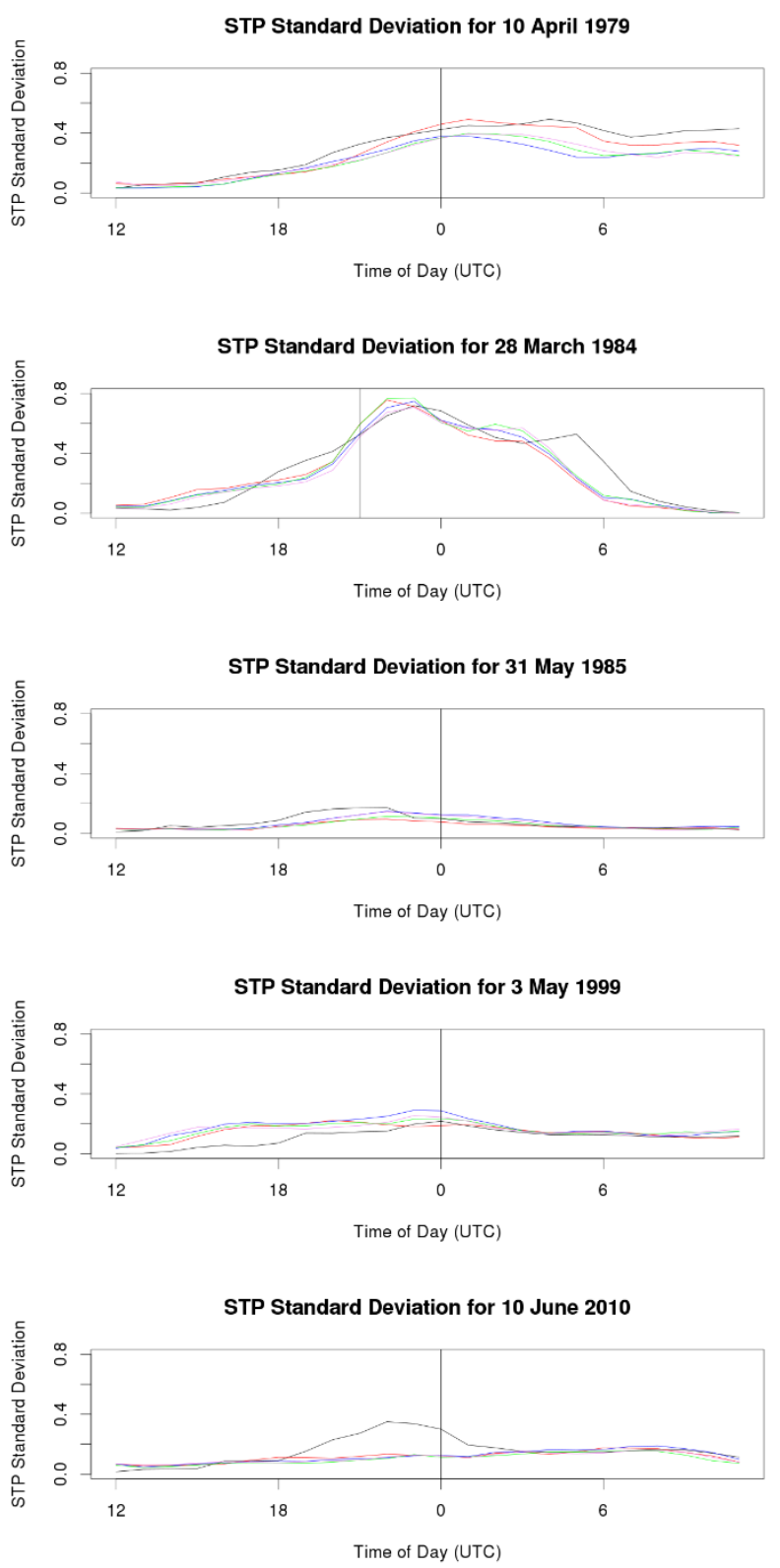

Figure 2. Standard Deviation (SD) statistics for all five tornadic outbreak cases in order of: April 10, 1979, March 28, 1984, May 31, 1985, May 3, 1999, and June 17, 2010 outbreak cases. Peak outbreak time is displayed by the vertical black line in each graph.

These results are not surprising as kinematic fields within tornado outbreaks are typically governed by the highly predictable synoptic-scale background flow patterns. Interestingly, the only major differences were observed in the 24-hour ensemble forecasts, suggesting predictability above 24-hours lead time remains consistent for tornado outbreak events. Thermodynamic indices exhibited more variability, but the general trend of 48-hour lead times and beyond performing consistently remained intact.

This research was conducted through the Blue Waters Student Internship Program which utilized the Blue Waters supercomputing center. Through this program, the student was exposed to highperformance computing research in the atmospheric sciences and gained many valuable educational lessons. For example, the student learned that scientific research requires flexibility in the research timeline. On numerous occasions timelines were altered to allow for unforeseen complications, including compilation issues, big data issues, and data transfers that took longer than originally anticipated.

Along with the computation and educational lessons learned, the student gained valuable experience in big data problems, which are becoming the standard in atmospheric science research, particularly research involving numerical weather prediction. In particular:

1. The student learned that computational resources must be modified at times to meet the needs of the research that is trying to be done. Due to a compilation issue, the student had to transfer all simulation output between an in-house computer and the Blue Waters system for postprocessing. This required tedious organization to ensure all cases were stored and post-processed properly and required some cases to be redone. The in-house computer was also a shared resource, which further hindered progress and required the student to be fully apprised of time and resource issues.

2. Time management played a huge role within this research, particularly accounting for run-times of some of the longer simulations. Many of the outbreak simulations took hours to run, so the student needed to set up distinct times for running these codes and a lot of time to transfer the data between an in-house computer and the Blue Waters computer. Additionally, transferring the cases for post-processing added an additional time component that was not foreseen at the beginning of this research.

3. The student gained valuable knowledge into the difficulties and limitations with tornadic outbreak modeling, as well as some of the meteorological advantages and disadvantages of diagnostic fields in outbreak research. While there are many convective indices that are utilized, and data can be collected for, not all of these indices are good at predicting such outbreaks at varying lead-time intervals.

Despite these computational challenges, this research would not have been able to have been completed without the computational power provide by the Blue Waters supercomputing center. Each simulation required roughly $16 \mathrm{~GB}$ of RAM, and each simulation produced roughly $20 \mathrm{~GB}$ of output after post-processing (leading to a final output size for all cases exceeding $2 \mathrm{~TB}$ ). The project would not have scaled well to systems at the student's university and would have required much longer than one year to complete, simply owing to the volume of simulations required.

\section{REFLECTIONS}

With any undergraduate research project, student inexperience with research and computing can increase the difficulty in a successful outcome. While the research done through this project was challenging at times, the student gained valuable experience with programming and high-performance computing, equipping the student for future research in big data and supercomputing, 
currently among the highest priorities in meteorological research. In particular, supercomputing has become highly prevalent in multiple public and private disciplines of atmospheric sciences. This research opportunity helped set the student apart from their peers by providing critical experience in a High-Performance Computing environment which will have further benefits as the student continues their education. The lessons and processes learned through this research will aid in the student's graduate studies beginning this fall. This research, in conjunction with the Blue Waters Student Internship Program and Summer Experience has given the student diligent training and preparedness for future graduate work and eventual career working with big data in atmospheric sciences.

\section{SUMMARY AND CONCLUSIONS}

It has been well established that tornadic outbreak events can be extremely difficult to forecast, especially several days in advance $[1,2,3]$. While efforts have been made to enhance such forecasts $[3,6,7,8,9,10]$, there is still room for growth within this field, particularly in medium-range outbreak forecasting. The primary goal of this research was to obtain an improved understanding of forecast uncertainty within tornado outbreaks at increasing lead times out to 5 days (considered medium-range forecasting). To accomplish this task, five major tornado outbreaks were simulated using stochastic perturbation ensembles at lead times out to 120 hours. Each of the five tornado outbreak cases chosen produced many tornadoes (over 20 per case), with many of them being violent EF2 or greater intensity tornadoes.

Once the test cases were chosen, utilizing input data from NARR, this data was run through ensemble simulations of WRF at 24, 48, 72,96 , and 120-hour lead time intervals. Each case and lead time included a ten-member stochastically perturbed ensemble to help generate (and thereby quantify) uncertainty in the 250 simulations. Post-processing of the data collected from WRF output files allowed many convective indices to be assessed using moment statistics, including MLCAPE, MLCINH, 0-3 km SRH, 0-1 km EHI, SCP, and STP.

Overall, thermodynamic convective indices seemed to show more variability across cases than index indices such as SCP, STP, and EHI. Moment statistics, such as standard deviation, show a much larger spread of data through thermodynamic convective indices like CAPE than with index indices like the three listed above. As shown in Figure 3, thermodynamic parameter such as CAPE show a bigger shift from the 24-hour forecast period to additional lead times, though the 48-hour to 120-hour lead times still behave similarly. This bigger shift from the 24-hour forecast to later lead times suggests outbreak predictability using CAPE alone is not sufficient, which has been well established in previous work [7].

The major finding in this study was that both thermodynamic and kinematic indices all behaved similarly at 48, 72, 96, and 120-hour lead times, regardless of the moment statistic selected. This was a surprising result given previous research, which typically noted a substantial increase in uncertainty after 72 hours lead time. This suggests outbreak predictability for these major events may not change significantly between 48 hours and 120 hours, which may give forecasters additional potential for predicting these events at longer lead times.

Furthermore, these results can be utilized in the future to continue drawing conclusions about forecasting techniques at short-term and medium-term ranges for tornado outbreaks. Future research is needed to ascertain the generalization of these results to all major tornado outbreak events. Some future work would include utilizing more test cases and more ensemble WRF forecasts to have more data to compare across tornadic outbreak cases. Additionally, previous research [13] has been done assessing medium-term forecast uncertainty of non-tornadic outbreak cases, and those results could be compared with the results of this study to gain a broader understanding of outbreak predictability across the full spectrum of outbreak events.
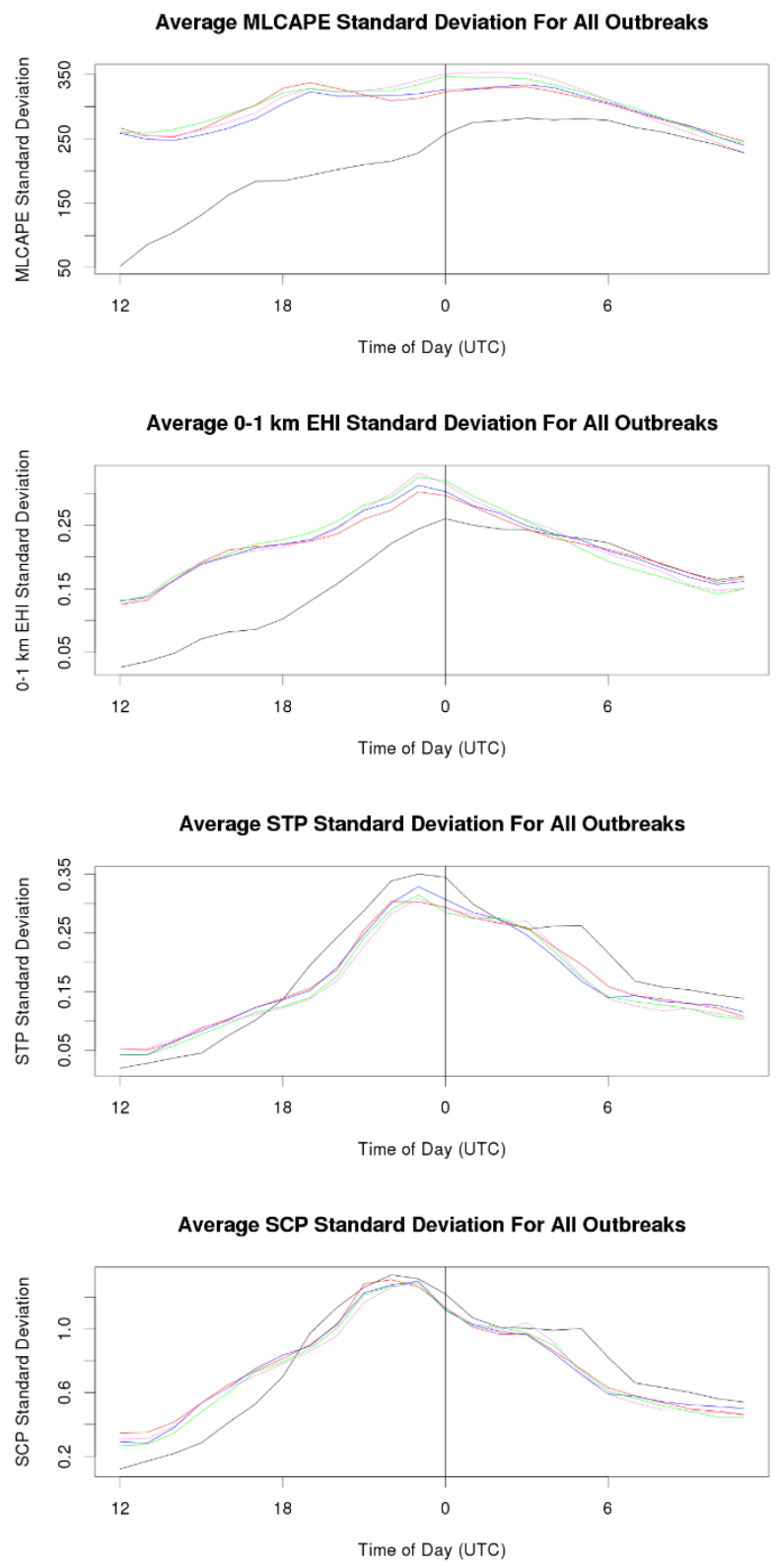

Figure 3. Average standard deviation (SD) statistics for one thermodynamic indices (MLCAPE) and for three kinematic indices (EHI, STP, and SCP).

\section{ACKNOWLEDGMENTS}

This research was funded through the Blue Waters sustainedpetascale computing project, which is supported by the National Science Foundation (awards OCI-0725070 and ACI-1238993) and the state of Illinois. This project was a result of the Shodor Blue Waters Undergraduate Student Internship Program and we are 
extremely thankful for their financial, technical, and educational support throughout the project.

\section{REFERENCES}

[1] Joseph G. Galway. 1977. Some Climatological Aspects of Tornado Outbreaks. Monthly Weather Review, 105, 477484.

[2] Russell S. Schneider, Joseph T. Schaefer, and Harold E. Brooks. 2004. Tornado outbreak days: An updated and expanded climatology (1875-2003). Preprints, 22nd Conf. on Severe Local Storms, Hyannis, MA, Amer. Meteor. Soc., P5.1.

[3] Chad M. Shafer, Andrew E. Mercer, Michael B. Richman, Lance M. Leslie, and Charles A. Doswell III. 2012. An assessment of areal coverage of severe weather indices for severe weather outbreak diagnosis. Weather and Forecasting, 27, 809-831.

[4] NOAA National Centers for Environmental Information (NCEI) National Climatic Data Center (NCDC). 2018. U.S. Tornado Climatology (1991-2010). Retrieved from https://www.ncdc.noaa.gov/climate-information/extremeevents/us-tornado-climatology.

[5] National Weather Service Office of Climate, Water, and Weather Services. 2018. NWS Weather Fatality, Injury and Damage Statistics. Retrieved from http://www.nws.noaa.gov/om/hazstats.shtml.

[6] Charles A. Doswell III and Jeffry S. Evans. 2003. Proximity sounding analysis for derechos and supercells: an assessment of similarities and differences. Atmospheric Research, 6768., 117-133.

[7] Andrew E. Mercer, Chad M. Shafer, Charles A. Doswell III, Lance M. Leslie, and Michael B. Richman. 2009. Objective classification of tornadic and nontornadic severe weather outbreaks. Monthly Weather Review, 137, 4355-4368.

[8] Andrew E. Mercer, Chad M. Shafer, Charles A. Doswell III, Lance M. Leslie, and Michael B. Richman. 2012. Synoptic composites of tornadic and nontornadic outbreaks. Monthly Weather Review. 140, 2590-2608.

[9] Chad M. Shafer, Andrew E. Mercer, Charles A. Doswell III, Michael B. Richman, and Lance M. Leslie. 2009. Evaluation of WRF forecasts of tornadic and nontornadic outbreaks when initialized with synoptic-scale input. Monthly Weather Review, 137, 1250-1271.

[10] Chad M. Shafer, Andrew E. Mercer, Lance M. Leslie, Michael B. Richman, and Charles A. Doswell III. 2010. Evaluation of WRF model simulations of tornadic and nontornadic outbreaks occurring in the spring and fall. Monthly Weather Review, 138, 4098-4119.

[11] Richard L. Thompson and Roger L. Edwards. 2000. An overview of environmental conditions and forecast implications of the 3 May 1999 tornado outbreak. Weather and Forecasting, 15, 682-699.

[12] Yussouf, N., D. C. Dowell, L. J. Wicker, K. H. Knopfmeier, and D. M. Wheatley. 2015. Storm-scale data assimilation and ensemble forecasts for the 27 April 2011 severe weather outbreak in Alabama. Monthly Weather Review, 143, 30443066 .

[13] Taylor Prislovsky and Andrew E. Mercer. 2017. Using Blue Waters to Assess Non-Tornadic Outbreak Forecast
Capability by Lead Time. The Journal of Computational Science Education, 8, 30-35, DOI:

https://doi.org/10.22369/issn.2153-4136/8/3/5.

[14] Storm Data. 2018. National Climatic Data Center, April 2018.

[15] NOAA National Severe Storms Laboratory (NSSL). 2018. May 3, 1999 Oklahoma/Kansas Tornado Outbreak. Retrieved from https://www.nssl.noaa.gov/about/history/may3rd/.

[16] NOAA National Weather Service Norman, OK WFO. 2018. The Red River Valley Tornado Outbreak of 1979. Retrieved from https://www.weather.gov/oun/events-19790410.

[17] NOAA National Weather Service State College, PA WFO. 2018. May 31, 1985 Tornado Outbreak. Retrieved from https://www.weather.gov/ctp/TornadoOutbreak_may311985.

[18] NOAA National Weather Service Twin Cities, MN WFO. 2018. Summary of June 17, 2010 Record Tornado Outbreak. Retrieved from https://www.weather.gov/mpx/20100617_MN_Record_Torn ado_Outbreak.

[19] NOAA National Weather Service Wilmington, NC WFO. 2018. Carolinas Tornado Outbreak: March 28, 1984. Retrieved from https://www.weather.gov/ilm/CarolinasOutbreak.

[20] Fedor Mesinger, Geoff Dimego, Eugenia Kalnay, Kenneth Mitchell, Perry C. Shafran, Wesley Ebisuzaki, Dusan Jovic, Jack Woolen, Eric Rogers, Ernesto H. Berbery, Michael B. Ek, Yun Fan, Robert Grumbine, Wayne Higgins, Hong Li, Ying Lin, Geoff Mankin, David Parrish, and Wei Shi. 2006. The North American Regional Reanalysis. Bulletin of the American Meteorological Society, 87, 343-360.

[21] Song-You Hong and Jeong-Ock Jade Lim. 2006. The WRF Single-Moment 6-Class Microphysics Scheme (WSM6). Journal of the Korean Meteorological Society, 42, 129-151.

[22] G. A. Grell and S. R. Freitas. 2014. A scale and aerosol aware stochastic convective parameterization for weather and air quality modeling. Atmos. Chem. Phys., 14, 5233-5250.

[23] Song-You Hong, Yign Noh, and Jimy Dudhia. 2006. A new vertical diffusion package with an explicit treatment of the entrainment processes. Monthly Weather Review, 134, 23182341.

[24] Eli J. Mlwaer, Steven J. Taubman, Patrick D. Brown, Michael J. Iacono, and Shepard A. Clough. 1997. Radiative transfer for inhomogeneous atmospheres: RRTM, a validated correlated-k model for the longwave. Journal of Geophysics Res., 102, 16663-16682.

[25] Jimy Dudhia. 1989. Numerical study of convection observed during the Winter Monsoon Experiment using a mesoscale two-dimensional model. Journal of Atmospheric Science, 46, 3077-3107.

[26] Richard L. Thompson, Roger Edwards, and Corey M. Mead. 2004. An update to the supercell composite and significant tornado indices. Retrieved from https://www.spc.noaa.gov/publications/thompson/stp_scp.pdf

[27] Judith Berner, G. J. Shutts, M. Leutbecher, and T. N. Palmer. 2009. A spectral stochastic kinetic energy backscatter scheme and its impact on flow-dependent predictability in the ECMWF ensemble prediction system. Journal of Applied Meteorology, 66, 603-62 